\title{
Impacto de la comunicación de funciones directivas sobre el compromiso de los trabajadores en la Administración Pública
}

\author{
Communication in Leadership Impact Commitment \\ of Workers in Public Administration
}

Recibido: abril 26 de 2013 | Aceptado: abril 12 de 2014 | Aceptado: agosto 12 de 2014

\author{
Miguel Ángel Mañas RodríGuez* \\ Carmen María Salvador Ferrer \\ Pedro Antonio Díaz Fúnez \\ Vicente Pecino Medina \\ Universidad de Almería, Almería, España
}

doi.org/10.11144/Javeriana.UPSY13-4.icfd

Para citar este artículo: Mañas, M. A., Salvador, C. M., Díaz, P. A., \& Pecino. V. (2014). Impacto de la comunicación de funciones directivas sobre el compromiso de los trabajadores en la Administración Pública. Universitas Psychologica, 13(4), 1581-1587. http://dx.doi.org/10.11144/Javeriana.UPSY13-4.icfd

* Correos electrónicos:marodrig@ual.es, cmsalva@ ual.es,pfunez@ual.es,vpecino@ual.es
RESUMEN

En este trabajo, se estudia la influencia que ejerce la comunicación de funciones directivas sobre el compromiso de los trabajadores, en diferentes equipos de la Administración Pública. Para esto, la muestra del presente trabajo fue de 355 empleados públicos. Se utilizó la escala de J. M. Peiró y V. González-Roma (1994) para analizar la comunicación de funciones directivas (alfa de Cronbach de 0.89) y la escala de O'Reilly y Chatman (1986) para analizar el compromiso de los trabajadores (alfa de Cronbach de 0.8). Los resultados indican que existen diferencias estadísticamente significativas en la comunicación de funciones directivas y el compromiso en función de los servicios. Además, se encontró que en algunos equipos el impacto que ejerce la comunicación de funciones directivas en el compromiso de los trabajadores es relevante.

Palabras clave

Compromiso organizacional; comunicación de funciones directivas; Administración Pública

\section{Abstract}

We study the influence of communication in leadership on workers' commitment in different teams in Public Administration. The sample of this study was 355 employees of Public Administration. The communication in leadership was evaluated with scale of Peiró and González-Roma (1994) (Cronbach's alpha of 0.89) and scale of O'Reilly and Chatman (1986) to analyze the commitment of workers (Cronbach's alpha of 0.8 ). The results indicate that there are significant differences in the communication of leadership and commitment in different teams. Also, in some teams, the communication of leadership impact significantly in commitment.

Keywords

Organizational commitment; communication in leadership; Public Administration 
Miguel Ángel Mañas Rodríguez, Carmen María Salvador Ferrer, Pedro Antonio Díaz Fúnez, Vicente Pecino Medina

\section{Introducción}

En la actualidad, se está inmerso en una situación de crisis donde cada vez se valora más el papel del líder (Rainey, 1999) y el compromiso de los trabajadores, lo que se observa conlleva a la estabilidad organizacional (Allen \& Meyer, 1990; Mañas, Salvador, Agulló, González, \& De Diego, 2007). Por consiguiente, parece que el compromiso organizacional es una variable fundamental en la organización. Del mismo modo, Pinillos (1996) señala que para la institución es muy importante la comunicación de funciones directivas. Resulta, entonces, imprescindible conocer estas influencias para poder explicar la conducta de los individuos, ya que la pertenencia a distintas formaciones supraindividuales con frecuencia no es casual (Cooley, Bond, \& Mao, 1981). Por tanto, en este estudio se centran los argumentos en la teoría de la influencia social (Festinger, 1950, 1954), según la cual los contextos pueden tener una influencia positiva o negativa sobre los individuos. Esta corriente postula que el contexto o grupo influye sustancialmente sobre las personas. Aún más, para que estas condiciones se presenten, resultan necesarios dos requisitos: primero, un consenso de los miembros del grupo sobre los aspectos sociales que son esenciales y segundo, una percepción colectiva sobre el grado de influencia (impacto) que ejercen dichos elementos en los individuos. Por tanto, en el ámbito organizacional existe cierta influencia del departamento u organización sobre los empleados y, además, es bastante probable que el líder ejerza influencias diferentes en cada una de las unidades departamentales. Así, pues, una vez expuestos los argumentos generales, se pasa a continuación a la delimitación de los términos centrales del presente estudio. Para ello, en primer lugar, la exposición estará centrada en la comunicación de funciones directivas y, posteriormente, se explicará el compromiso organizacional.

\section{Comunicación de funciones directivas}

La modernización de las organizaciones tanto públicas como privadas y su adaptación a la denominada sociedad de la información y del conocimiento fomentan el diseño de estructuras organizacionales cada vez más planas y con menos niveles jerárquicos (Peiró, 1990). Organizaciones donde no solo se valora asumir las responsabilidades propias del puesto, sino la capacidad de tomar decisiones en situaciones de incertidumbre y el carácter emprendedor en roles cada vez más complejos. Esta complejidad supone que para conseguir un rendimiento superior, el individuo tendría que superar los requerimientos mínimos o tareas definidas en la descripción del puesto, en aras a la consecución de las metas de la organización. Para tal finalidad resulta esencial la comunicación de las funciones directivas.

La comunicación se podría definir como el intercambio de información entre el emisor (líder) y el receptor (subordinados), así como la interferencia (percepción) de significado entre ellos (Bowditch \& Buono, 1997). El análisis de este intercambio revela que la comunicación es un proceso bidireccional de elementos vinculados de forma consecutiva. En un sentido más genérico, la comunicación se puede considerar como uno de los procesos básicos de cualquier sistema. Siguiendo esta línea, la comunicación podría ser catalogada como un proceso dinámico fundamental para la existencia, el crecimiento, el cambio y la conducta de todos los seres humanos (Thayer, 1975).

En esta misma línea, adquiere sentido defender que a través de la comunicación las personas establecen relaciones interpersonales funcionales que les permiten trabajar de forma conjunta y alcanzar las metas previstas. Por consiguiente, parece lógico pensar que el proceso comunicativo no es sencillo y que, además, en él interviene un conjunto heterogéneo de elementos. Por consiguiente, la comunicación es imprescindible para el buen funcionamiento de cualquier organización, ya que ayuda a mantenerla unida, proporcionando la información necesaria para la realización de las actividades requeridas. Se trata, pues, de uno de los elementos conductores para aunar los esfuerzos de todas las personas de la organización en la dirección adecuada, así como para llevar a cabo las metas, pudiendo cumplir con los objetivos propuestos. Por ello, la comunicación dentro de la organización es una de 
las variables más importantes para poder dirigirla a la excelencia y la calidad (Mañas, 2005). Tal como apunta Pinillos (1996), este instrumento sería de gran utilidad para optimizar el funcionamiento de la organización, sobre todo si se tiene presente que resulta de gran utilidad en la cohesión de la plantilla, fomentando sentimiento de pertenencia y tornando a la empresa más competitiva. En resumidas cuentas, según apunta Pinillos, la comunicación de las funciones directivas es básica en la eficacia organizacional.

Además, tal como indican Westphalen y Piñuel (1993), cuando dentro de la empresa existe una excelente comunicación de las funciones directivas, los trabajadores tienden a manifestar una actitud positiva hacia el trabajo, contribuyendo, en mayor medida, al beneficio global de la organización. En este sentido, Rhee (2008), en una investigación realizada con profesores, muestra cómo la comunicación de funciones directivas es básica para que exista el compromiso. Aún más, a lo largo de su investigación, contempla que las funciones directivas de los profesores son esenciales en el compromiso de los usuarios. Del mismo modo, otros autores (Aldoory, 2001; Grunig \& Huang, 2000) apuestan por la comunicación como herramienta básica para conseguir el compromiso.

\section{Compromiso organizacional}

Tal como recogen Mañas, Salvador, Boada, González y Agulló (2007), la evidencia empírica indica que la estabilidad de la organización se consigue si las instituciones logran comprometer a sus empleados. En este sentido, en el modelo inicial propuesto por Meyer y Allen (1984) se distingue entre el compromiso afectivo y el compromiso de continuidad. El primero se refiere a la identificación y la implicación emocional del individuo con los valores de la organización. Por su parte, el segundfo se basa en los costes personales que supondría para individuo abandonar la organización. Un tiempo más tarde, estos autores definen el compromiso normativo, conceptualizado como la obligación moral que sienten los trabajadores de permanecer en la institución (Allen \& Meyer, 1990). De modo global, podría definirse como la obligación moral que siente el trabajador de permanecer en la empresa.

Por consiguiente, el compromiso podría ser definido como "la fuerza que brinda a los individuos su curso de acción" (Meyer \& Herscovitch, 2001, p. 308). Además, según establecen O’Reilly y Chatman (1986), "las raíces del compromiso están en la indentificación y la internalización se basa en las conductas pro-sociales" (p. 493). Las investigaciones recientes demuestran que el compromiso de los trabajadores es esencial y, al mismo tiempo, que se trata de un constructo multidimensional (Meyer \& Allen, 1997; Meyer, Stanley, Herscovitch, \& Topolnytsky, 2002). En concreto, el compromiso de los trabajadores podría ser un indicador del grado de identificación del individuo con el equipo (Costa \& Anderson, 2011). En línea con estos argumentos, se encuentran los postulados de Bishop y Scott (2000), quienes establecen que el compromiso del equipo se caracteriza porque: (1) es una fuerte creencia en la aceptación de las metas y los valores de la organización, (2) el individuo se esforzará para conseguir los intereses del equipo y (3) se establecen esfuerzos personales por seguir manteniendo la identidad del equipo.

Por su parte, Manz y Sims (1993) señalan que la fuerza del equipo está altamente relacionada con el compromiso de sus miembros. Aún más, Costa y cols. (2001) demuestran que la credibilidad del equipo se relaciona con el compromiso. De igual forma, convendría señalar que algunos estudios (Brockner, Siegel, Daly, Tyler, \& Martin, 1997; Cook \& Wall, 1980) reflejan que el compromiso es esencial, dado que incrementa los efectos positivos de la institución, por ejemplo, los resultados obtenidos y la imagen social mejoran la cooperación entre los miembros de la institución y optimizan el clima organizacional. En este sentido, tal como reflejan Costa y Anderson (2011), el compromiso es una variable esencial en el equipo. Dado que la dirección ejerce un papel básico dentro de la empresa, el rol del dirigente podría ser la clave para el compromiso. No obstante, tal como indican Brunetto y Farr Warthon (2004), las habilidades comunicativas de los gerentes resultan esenciales para conseguir el apego de los empleados a la organización. En este 
sentido, tal como señalan estos autores, parece que la investigación ha centrado poco interés en este tema. Es precisamente en este punto donde nosotros depositamos el interés de nuestro trabajo.

En síntesis, tal como ha quedado demostrado, tanto la comunicación de las funciones directivas como el compromiso de los trabajadores parecen ser elementos esenciales dentro de la organización. Por consiguiente, el objetivo del presente estudio consiste en comprobar si la comunicación de funciones directivas tiene algún tipo de influencia sobre el compromiso en los trabajadores de una Administración Pública en las distintas unidades de trabajo.

Para analizar, detalladamente, este objetivo de trabajo, se establecen dos hipótesis que se presentan a continuación:

Hipótesis 1. La comunicación de funciones directivas y el compromiso organizacional mostrarán diferencias estadísticamente significativas según los distintos servicios.

Hipótesis 2. La comunicación de funciones directivas influye significativa y positivamente en el compromiso organizacional de los trabajadores.

\section{Método}

\section{Procedimiento}

La obtención de datos se realizó mediante la cumplimentación de un cuestionario compuesto por preguntas relacionadas con las variables objeto del estudio. A fin de facilitar la participación y poder responder el cuestionario, se facilitó tiempo dentro del horario laboral. Además, los miembros del equipo de investigación estaban presentes para aclarar cualquier duda que pudiera surgir. Los cuestionarios recogían información demográfica (sexo, edad, estado civil), así como las preguntas correspondientes a las variables. La cumplimentación de los cuestionarios fue voluntaria y totalmente confidencial.

\section{Muestra}

Este estudio se llevó a cabo con 355 trabajadores de la Administración Pública, nótese que en este trabajo se excluyeron aquellos servicios que no contaban con más de 10 trabajadores (esto supone un 39.2\% del valor muestral total). Así pues, el personal estaba formado por el Servicio de Residencia Asistida (15\%), Servicio de Vías Provinciales (8.8\%), Servicio de la Mujer (7\%), Servicio de Recursos Humanos y Régimen del Interior (6.6\%), Servicio Jurídico-Administrativo (6.2\%), Servicio de Deportes y Cultura (6.2\%), Servicio de Cooperación Local (5.7\%) y Servicio de Cooperación Provincial (5.3\%). En relación con la variable sexo, el $49.6 \%$ de la muestra estaba formada por hombres, frente al $50.4 \%$ de mujeres. Un $72 \%$ señalaba estar casado o vivir en pareja, frente a un $22 \%$ que estaba soltero y un $5 \%$ divorciado.

\section{Instrumentos de medida}

La comunicación de funciones directivas fue medida a través de una escala desarrollada por Peiró y González-Roma (1994), basándose en la taxonomía de Yukl y Van Fleet (1992) sobre conductas directivas. En concreto, la escala está conformada por tres ítems que hacen referencia al grado de comunicación que tiene el jefe o superior inmediato en relación con la transmisión de información referente a políticas y prácticas de la organización. El formato de respuesta de las preguntas es tipo Likert con un intervalo de respuesta de 5 puntos, siendo 1 muy en desacuerdo y 5 muy de acuerdo (por ejemplo, "Nos informa de las políticas y prácticas de la organización"), siendo el coeficiente alpha de Cronbach 0.89.

La escala de compromiso se midió mediante 3 ítems extraídos y adaptados del cuestionario de compromiso organizacional elaborado por O'Reilly y Chatman (1986). En concreto, el compromiso en esta escala se define como la fuerza con la que una persona se identifica con la organización. Los ítems se contestan utilizando una escala de cinco alternativas de respuesta que oscilan entre $1=$ muy en desacuerdo y $7=$ muy de acuerdo (ejemplo: "Me siento orgulloso cuando les digo a otros en que equipo trabajo"). El coeficiente alfa de Cronbach correspondiente al valor general de la escala es de 0.8 . 


\section{Análisis}

Los datos, se sometieron a análisis en el programa SPSS 18. Para contrastar las hipótesis de trabajo, se realizaron diferentes análisis: descriptivos, de correlación de Pearson y ANOVA de un factor (Hipótesis 1) y, por otro lado, análisis de regresión (Hipótesis 2).

\section{Resultados}

\section{Análisis descriptivos, correlaciones y ANOVA de un factor (Servicios)}

Se harán primero unos análisis de las variables empleadas: descriptivos (media y desviación estándar), de correlación de Pearson y de varianza (ANOVA de un factor-servicios-) (Tabla 1).

Así, en los resultados obtenidos en las puntuaciones medias de ambos constructos, se encuentra un valor de 3.42 (desviación estándar de 1.15) en el caso de la comunicación de funciones directivas y una puntuación de 5.28 asociada a la variable de compromiso organizacional (desviación estándar de 0.97). En lo que respecta al análisis de correlación de sendos términos (comunicación de funciones directivas y compromiso), se obtuvo una puntuación de $r=0.189 p>0.001$. Finalmente, con el propósito de hacer un análisis preliminar para conocer si hay variaciones entre grupos en ambos términos, se realizó un análisis de varianza de un factor (servicios). En este sentido, los datos indican que existen diferencias estadísticamente significativas en la comunicación de funciones directivas ( $F$ $=2.35 ; p>0.001$ ).

\section{Análisis de regresión}

En lo que respecta a la Tabla 2 (Media cuadrática =9.98; $F=11.064$; Significación $=0$ ), es decir, al impacto de la comunicación de funciones directivas sobre el compromiso organizacional ( $\beta$ $=0.162$; Sig. $=0.001$ ), en primer lugar se observa que este es determinante en el compromiso a nivel general, o sea, sin establecer diferencias por servicios.
Posteriormente, se realizó este análisis, estableciendo diferencias por servicios. Los datos reflejaron que no en todos los servicios valorados es significativo el impacto de la comunicación de funciones directivas en el compromiso de los trabajadores. En concreto, se hallaron resultados estadísticamente significativos en el Servicio de Vías Provinciales (Tabla 3) (Media cuadrática $=4.76 ; F=8.75$; Significación $=0.002, \beta=0.455$; Sig. $=0.009)$ y el Servicio de Deportes y Cultura (Tabla 4) (Media cuadrática $=3.86 ; F=20.178 ;$ Significación $=$ $0.02 ; \beta=0.79 ;$ Sig. $=0.021$ ).

Se concluye, pues, resaltando que existen diferencias entre servicios en la percepción del compromiso y que este se ve afectado por la comunicación de funciones directivas. No obstante, convendría señalar que este impacto no es significativo en todos los servicios analizados.

\section{Discusión}

Analizando los datos de la Administración Pública, se encuentra que las hipótesis del estudio se cumplen. En concreto, en la primera hipótesis se pronosticó que el compromiso y la comunicación de funciones directivas mostrarán diferencias estadísticamente significativas según los servicios. Pues bien, los datos obtenidos mediante análisis de varianza confirman estos planteamientos.

Por otro lado, en la segunda hipótesis, donde se predecía que la comunicación de funciones directivas influye significativa y positivamente en el compromiso de los trabajadores, también se confirma. En concreto, los datos demuestran que dicho impacto no se refleja en todos los servicios valorados. Se concluye, pues, señalando que la comunicación de funciones directivas tiene un notable impacto en el compromiso de los trabajadores de la Administración Pública.

Estos hallazgos confirman que ciertos aspectos correspondiente a los roles directivos, en concreto, la comunicación de funciones directivas, parece influir en el compromiso de los trabajadores (Brunetto \& Farr-Wharton, 2004). Sería conveniente que en futuros estudios se resalte la necesidad de que los investigadores se esfuercen por incluir estas 
variables dentro de sus intereses, adoptando nuevas perspectivas de análisis, resultando importante adaptar los modelos y las técnicas para ajustarlos a las exigencias del entorno (González \& Peiró, 1992). Estos datos vienen a confirmar parcialmente los postulados establecidos por Costa y Anderson (2011), quienes ponen de manifiesto, a través de un análisis multinivel, en qué medida el compromiso es una variable que puede verse afectada por el contexto organizacional. En el presente caso, se utilizaron instrumentos metodológicos más simples, pero, a nuestro entender, se ha avanzado ligeramente en estos argumentos, pues se señaló que la comunicación de funciones directivas tendría que ser considerada en la estabilidad de los trabajadores.

En este sentido, es preciso señalar que el compromiso de los trabajadores de la Administración Pública presenta variaciones interservicios. Además, que parte de esta varianza se puede explicar por la comunicación de funciones directivas. Los autores piensan que estos argumentos encuentran sus sustentos en la literatura previa (Brunetto \& Farr Warthon, 2004). Se cree que esto es el principal aspecto del estudio, dado que, tal como señala Cohen (2003), en un metaanálisis, el compromiso se relaciona con múltiples variables (satisfacción, motivación, rotación, entre otras), pero todas al mismo nivel, o sea, a nivel individual.

Por ende, se entiende que con esta investigación se evidencia la relevancia de estudiar la influencia del contexto sobre las variables individuales. Se podría, por tanto, resaltar los siguientes principios de la teoría de la influencia social, en concreto: el grupo parece que es interdependiente y, además, podrían existir ciertos aspectos organizacionales que influyen en los individuos. En el presente caso, la comunicación de funciones directivas influye en el compromiso de los trabajadores.

Como limitación de este trabajo, y dado quese encontraron resultados significativos en algunos servicios donde la comunicación de funciones directivas parece una variable esencial, se cree necesario destacar el interés en el perfil de los grupos de la organización. De igual forma, podría resultar interesante conocer, a través de cross level (multinivel), aquellos aspectos que llevan a los grupos a establecer consenso dentro de los equipos. Asimismo, convendría realizar estudios longitudinales y aplicar las herramientas a organizaciones diferentes de la Administración Pública.

En cuanto a las implicaciones prácticas, se podría decir que es el primer paso que demuestra el impacto que parece ejercer la comunicación de funciones directivas en el compromiso de los trabajadores. Por lo tanto, los directivos de las organizaciones tienen un papel importante en ese compromiso. En síntesis, comunicar el plan de actuación de la empresa afecta el compromiso de los trabajadores de la Administración Pública; luego comunicar en el presente lo que los gerentes pretenden hacer en el futuro (Kotler, Keller, Brady, Goodman, \& Handsen, 2009) podría ser la "nueva clave para el éxito".

\section{Referencias}

Aldoory, L. (2001). Making health connections meaningful for women: Factors that influence involvement. Journal of Public Relations Research, 13(2), 163-185.

Allen, N. J., \& Meyer, J. P. (1990). The measurement and antecedents of affective, continuance and normative commitment to the organization. Journal of Occupational and Organizational Psychology, 63(1), 1-18.

Bishop, J. W., \& Scott, K. D. (2000). An examination of organizational and team commitment in a selfdirected team environment. Journal of Applied Psychology, 85(3), 439-450.

Bowditch, J. L., \& Buono, A. F. (1997). A primer on organizational behavior (4.a ed.). New York: JohnWiley \& Sons.

Brockner, J., Siegel, P. A., Daly, J. P., Tyler, T., \& Martin, C. (1997). When trust matters: The moderating effects of outcome favorability. Administrative Science Quarterly, 42(3), 558-583.

Brunetto, Y., \& Farr-Wharton, R. (2004). Does the talk affect your decision to walk: A comparative pilot study examining the effect of communication practices on employee commitment post-managerialism. Management Decision, 42 (3-4), 579-600.

Cohen, A. (2003). Multiple commitments in the workplace: An integrative approach. Mahwah, NJ: Erlbaum.

Cook, J., \& Wall, T. (1980). New work attitude measures of trust, organizational commitment and perso- 
nal need non-fulfilment. Journal of Occupational and Organizational Psychology, 53(1), 39-52. http:// dx.doi.org/10.1111/j.2044-8325.1980.tb00005.x

Cooley, W. W., Bond, L., \& Mao, B. J. (1981). Analyzing multi-level data. En R. Berk (Ed.), Educational evaluation methodology: The state of the art (69-83). Baltimore: The Johns Hopkins University Press.

Costa, A., \& Anderson, N. (2011). Measuring trust in teams: Development and validation of a multifaceted measure of formative and reflective indicators of team trust. European Journal of Work and Organizational Psychology, 20(1), 119-154.

Festinger, L. (1950). Informal social communication. Psychological Review, 57(5), 271-282.

Festinger, L. (1954). A theory of social comparison processes. Human Relations, 7(2), 117-140.

González, V., \& Peiró, J. (1992). Técnicas de investigación multinivel en las organizaciones. En M. Clemente (Ed.), Psicología social. Métodos y técnicas de investigación (pp. 349-365). Madrid: Eudema.

Grunig, J. E., \& Huang, Y.H. (2000). From organizational effectiveness to relationship indicators: Antecedents of relationships, public relations strategies, and relationship outcomes. En J. A. Ledingham \& S. D. Bruning (Eds.), Public relations as relationship management: A relational approach to the study and practice of public relations (pp. 23-53). Mahwah, NJ: Erlbaum.

Kotler, P., Keller, R., Brady, M., Goodman, M., \& Handsen, T. (2009). Marketing

management. Essex, UK: Pearson.

Mañas, M. A. (Dir.). (2005). La comunicación interna. Experiencias en una Administración Pública. Almería: Instituto de Estudios Almerienses.

Mañas, M. A., Salvador, C., Agulló, J., González, E., \& De Diego, R. (2007). La satisfacción y el bienestar psicológico como antecedentes del compromiso organizacional. Psicothema, 19(3), 395-400.

Manz, C. C., \& Sims, H. P. (1993). Business without bosses: How self-managing teams are building highperformance companies. Nueva York: Wiley.

Meyer, J. P., \& Allen, N. J. (1997). Commitment in the workplace: Theory, research, and application. Thousand Oaks, CA: Sage.

Meyer, J.P., \& Allen, N.J. (1984). Testing the "sidebet theory" of organization commitment: Some methodological considerations. Journal of Applied Psychology, 69, 372-378.

Meyer, J. P., \& Herscovitch, L. (2001). Commitment in the workplace: Toward a general model. Human Resource Management Review, 11(3), 299-326.

Meyer, J. P., Stanley, D. J., Herscovitch, L., \& Topolnytsky, L. (2002). Affective, continuance, and normative commitment to the organization: A meta-analysis of antecedents, correlates, and consequences. Journal of Vocational Behavior, 61(1), 20-52.

O'Reilly, C., \& Chatman, J. (1986). Organizational commitment and psychological attachment: The effects of compliance, identification, and internalization on prosocial behavior. Journal of Applied Psychology, 71(3), 492-499.

Peiró, J. M. (1990). Las nuevas tecnologías en organización. Nuevas perspectivas psicosociales. Barcelona: Promociones Publicaciones Universitarias S. A.

Peiró, J. M., \& González-Roma, V. (Dirs.). (1994). Estudio sobre los correlatos psicológicos del absentismo en el personal del Servicio Valenciano de Salud: calidad de vida en las instituciones sanitarias (Proyecto de investigación). Valencia: Unidad de Investigación de Psicología de las Organizaciones y del Trabajo y de los Recursos Humanos/Universitàt de Valencia.

Pinillos, J. L. (1996). La mentalidad postmoderna. Psichothema, 8(1), 229-240.

Rainey, H. (1999). Using comparisons of public and private organizations to assess innovative attitudes among members of organizations. Public Productivity and Management Review, 23(2), 130-149.

Rhee, Y. (2008). Risk communication management: A case study on Brookhaven National Laboratory. Journal of Communication Management, 12(3), 224-242.

Thayer, L. (1975). Comunicación y sistemas de comunicación. Barcelona: Península.

Westphalen, M. H., \& Piñuel, J. L. (1993). La dirección de la comunicación. Madrid: Ediciones del Prado.

Yukl, G., \& Van Fleet, D. D. (1992). Theory and research on leadership in organizations. En M. D. Dunnette \& L. M. Hough (Eds.), Handbook of industrial and organizational psychology (Vol. 3, 2.a ed., pp. 147-197). Palo Alto, CA: Consulting Psychologists Press. 
